\title{
SOME CONSIDERATIONS ON ELECTRONIC ENERGY OF ITINERANT-ELECTRON METAMAGNETIC MATERIALS WITH VOLUME-DEPENDENT EXCHANGE ENERGY
}

\author{
M. A. GRADO-CAFFARO*, M. GRADO-CAFFARO \\ and S. L. SAPIENZA ${ }^{\dagger}$ \\ C/Julio Palacios 11, 9-B, 28029-Madrid, Spain
}

(Received 5 December 2000; In final form 19 March 2001)

\begin{abstract}
Electronic energy of itinerant-electron metamagnetic materials is examined by taking into consideration volume-dependent exchange energy between up and down spin electrons. In particular, the average value of the electronic energy is formulated in terms of the energy bandwidth which is closely related to volume; from the above average value, the average value of the exchange energy may be evaluated.
\end{abstract}

Keywords: Electronic energy; Itinerant-electron metamagnetism; Exchange energy; Averaged electronic energy; Energy bandwidth

\section{INTRODUCTION}

In the context of itinerant-electron metamagnetism, energy is divided into electronic energy and lattice energy; lattice energy depends upon volume variation while electronic energy is assumed to be volume-independent in a considerable part of the literature. For instance, in Ref. [1] exchange energy between up and down spin

${ }^{*}$ Corresponding author.

${ }^{\dagger}$ Scientific consultant. 
electrons is regarded, for simplicity, as independent of volume; therefore, by taking into account that electronic energy depends on exchange energy (Stoner model for $\mathrm{T}=0^{\circ} \mathrm{K}$ ), it is obvious that, in Ref. [1], volume-independent electronic energy is assumed. However, more accurate assumptions can be made in order to achieve certain goals representing a better understanding of the involved phenomenology. With respect to this, the aim of this paper is to consider volume variation of the exchange energy through the energy bandwidth since there exists a close relation between bandwidth and volume $[2,3]$.

\section{THEORETICAL CONSIDERATIONS}

We begin by writing down the expression for the electronic energy at $\mathrm{T}=0^{\circ} \mathrm{K}$ of an itinerant-electron metamagnetic system in the context of the Stoner model, namely $[1,3,4]$ :

$$
E_{e}(W)=\int_{-W}^{E_{F 1}} E g(E, W) d E+\int_{-W}^{E_{F 2}} E g(E, W) d E-\frac{1}{4} M^{2} J(W)-\mu_{B} M H
$$

where $2 W$ is energy bandwidth, $E$ denotes energy, $g(E)$ is the electronic density of states, $E_{F 1}$ and $E_{F 2}$ are the Fermi levels of the up and down spin bands respectively, $J$ is the exchange energy between up and down spin electrons, $\mu_{\mathrm{B}}$ is the Bohr magneton, $\mathbf{M}$ is magnetization and $H$ stands for an applied magnetic field. On the other hand, we can write [2]:

$$
\mathrm{W}=\mathrm{W}_{\mathrm{o}} \exp \left[-\alpha\left(\mathrm{V}-\mathrm{V}_{\mathrm{o}}\right) / \mathrm{V}_{\mathrm{o}}\right]
$$

where $\mathrm{V}$ denotes volume, $\mathrm{V}_{\mathrm{o}}$ is the initial volume, $\mathrm{W}_{\mathrm{o}}$ is the value of $\mathrm{W}$ when $\mathrm{V}=\mathrm{V}_{\mathrm{o}}$, and $\alpha$ is a parameter such that $1 \leq \alpha \leq 5 / 3$. For $\mathrm{V} \gg \mathrm{V}_{\mathrm{o}}$ (theoretically as $\mathrm{V} \rightarrow \infty$ ) we have that $\mathrm{W} \rightarrow \mathrm{O}$; J depends upon $\mathrm{V}$ and, by taking into account Eq. (2), it is evident that $\mathrm{J}$ depends upon W. Then, by considering expression (1), we formulate 
the following average value:

$$
\begin{aligned}
\left\langle E_{\mathrm{e}}\right\rangle \equiv & \frac{1}{-\mathrm{W}_{\mathrm{o}}} \int_{\mathrm{W}_{\mathrm{o}}}^{0} \mathrm{E}_{\mathrm{e}}(\mathrm{W}) \mathrm{dW} \\
= & \frac{1}{\mathrm{~W}_{\mathrm{o}}} \int_{\mathrm{o}}^{\mathrm{W}_{\mathrm{o}}} \mathrm{dW} \int_{-\mathrm{W}}^{\mathrm{E}_{\mathrm{F} 1}} \mathrm{Eg}(\mathrm{E}, \mathrm{W}) \mathrm{dE} \\
& +\frac{1}{\mathrm{~W}_{\mathrm{o}}} \int_{\mathrm{o}}^{\mathrm{W}_{\mathrm{o}}} \mathrm{dW} \int_{-\mathrm{W}}^{\mathrm{E}_{\mathrm{F} 2}} \mathrm{Eg}(\mathrm{E}, \mathrm{W}) \mathrm{dE} \\
& -\frac{1}{4} \cdot \frac{\mathbf{M}^{2}}{\mathrm{~W}_{\mathrm{o}}} \int_{\mathrm{o}}^{\mathrm{W}_{\mathrm{o}}} \mathrm{J}(\mathrm{W}) \mathrm{dW}-\frac{\mu_{\mathrm{B}} \mathrm{MH}}{\mathrm{W}_{\mathrm{o}}} \int_{0}^{\mathrm{W}_{\mathrm{o}}} \mathrm{dW}
\end{aligned}
$$

so that from expression (3) it follows:

$$
\begin{aligned}
\int_{0}^{W_{0}} J(W) d W= & -\frac{4 W_{0}}{M}\left(\frac{\left\langle E_{e}\right\rangle}{M}+\mu_{B} H\right) \\
+ & \frac{4}{M^{2}}\left[\int_{0}^{W_{0}} d W \int_{-W}^{E_{F 1}} E g(E, W) d E\right. \\
& \left.\quad+\int_{0}^{W_{0}} d W \int_{-W}^{E_{F 2}} E g(E, W) d E\right]
\end{aligned}
$$

Equation (4) provides a way to estimate the $\mathrm{W}$-variation of $\mathrm{J}$ so that we can say that $J(W)$ is a function such that its average value is $W_{0}^{-1}$ times the right-hand side of (4); rewriting this relationship, it is easy to see that:

$$
\begin{aligned}
\int_{0}^{W_{0}} J(W) d W= & -\frac{4 W_{o}}{M}\left(\frac{\left\langle E_{e}\right\rangle}{M}+\mu_{B} H\right) \\
& +\frac{4}{M^{2}}\left[\iint_{\Omega_{1}} E g(E, W) d E d W\right. \\
& \left.+\iint_{\Omega_{2}} E g(E, W) d E d W\right]
\end{aligned}
$$

where $\Omega_{1}$ and $\Omega_{2}$ are the integration domains (depicted in Fig. 1) corresponding to the involved double integrals.

On the other hand, it is important to point out that an elliptic electronic density of states is usually employed, namely (see, for 


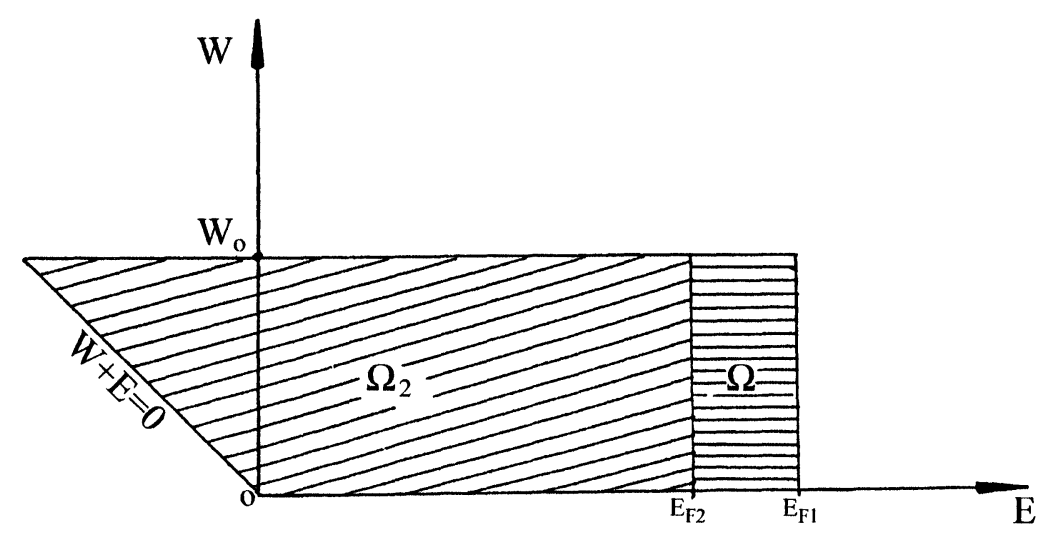

FIGURE 1 Integration domains $\Omega_{1}$ and $\Omega_{2}\left(\Omega_{1}=\Omega_{2} \cup \Omega\right)$.

example, Refs. [1, 3]):

$$
\mathrm{g}(\mathrm{E}, \mathrm{W}) \approx 3 \mathrm{~W}^{-2}\left(\mathrm{~W}^{2}-\mathrm{E}^{2}\right)^{1 / 2}
$$

However, from a strictly quantitative point of view, expression (6) does not agree well with experiment; only a certain qualitative agreement with experimental data takes place. At any rate, to date there are no rigorous theories for the density of states that agree with experiment (for all metamagnetic materials). Then, to some extent, Eq. (6) is somewhat satisfactory for certain materials excluding intermetallic compounds. On replacing (6) into (5), an acceptable estimation of the $\mathrm{W}$-variation of the exchange energy is feasible.

\section{CONCLUSIONS}

The preceding discussion offers a strategy to evaluate the bandwidth variation of the exchange energy between up and down spin electrons. In the above discussion, the electronic density of states plays an important role; it is clear that $\mathrm{W}$ acts as a parameter in the integrals involved in formula (1) so that $E_{e}$ becomes a function of $W$. On the other hand, it is interesting to notice that the average value $\langle J\rangle \equiv$ $\mathrm{W}_{\mathrm{o}}^{-1} \int_{0}^{\mathrm{W}_{\mathrm{o}}} \mathrm{J}(\mathrm{W}) \mathrm{dW}$ constitutes a useful quantity which can be evaluated by means of expression (5). 


\section{References}

[1] Duc, N. H., Givord, D., Lacroix, C. and Pinettes, C. (1992). Europhys. Lett., 20 , $47-52$.

[2] Slater, J. C. and Koster, G. (1954). Phys. Rev., 94, 1498.

[3] Grado-Caffaro, M. A. and Grado-Caffaro, M. (1994). Act. Pass. Electronic Comp., $17,177-178$.

[4] Grado-Caffaro, M. A. and Grado-Caffaro, M. (1997). Act. Pass. Electronic Comp., 20, 91-94. 

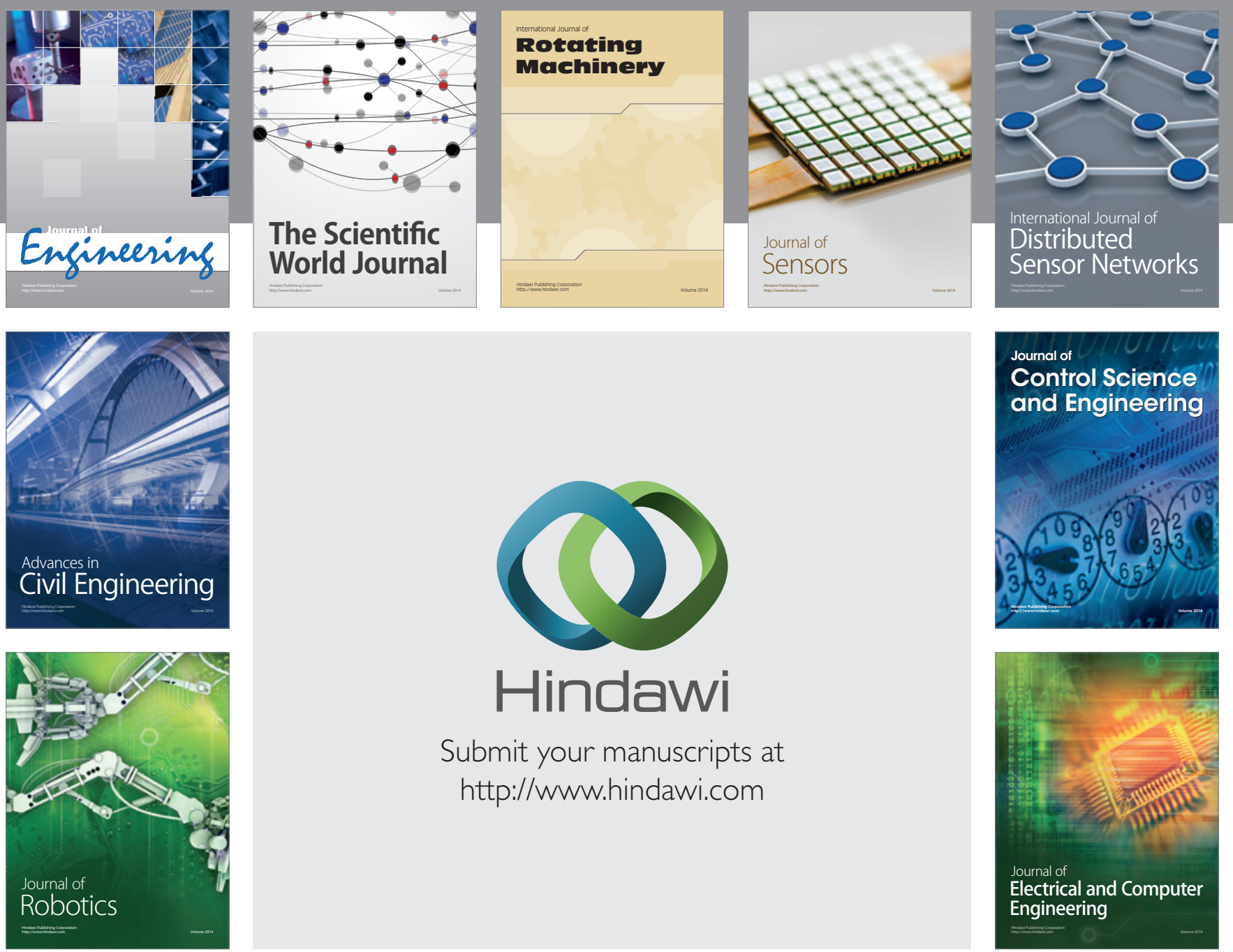

Submit your manuscripts at

http://www.hindawi.com
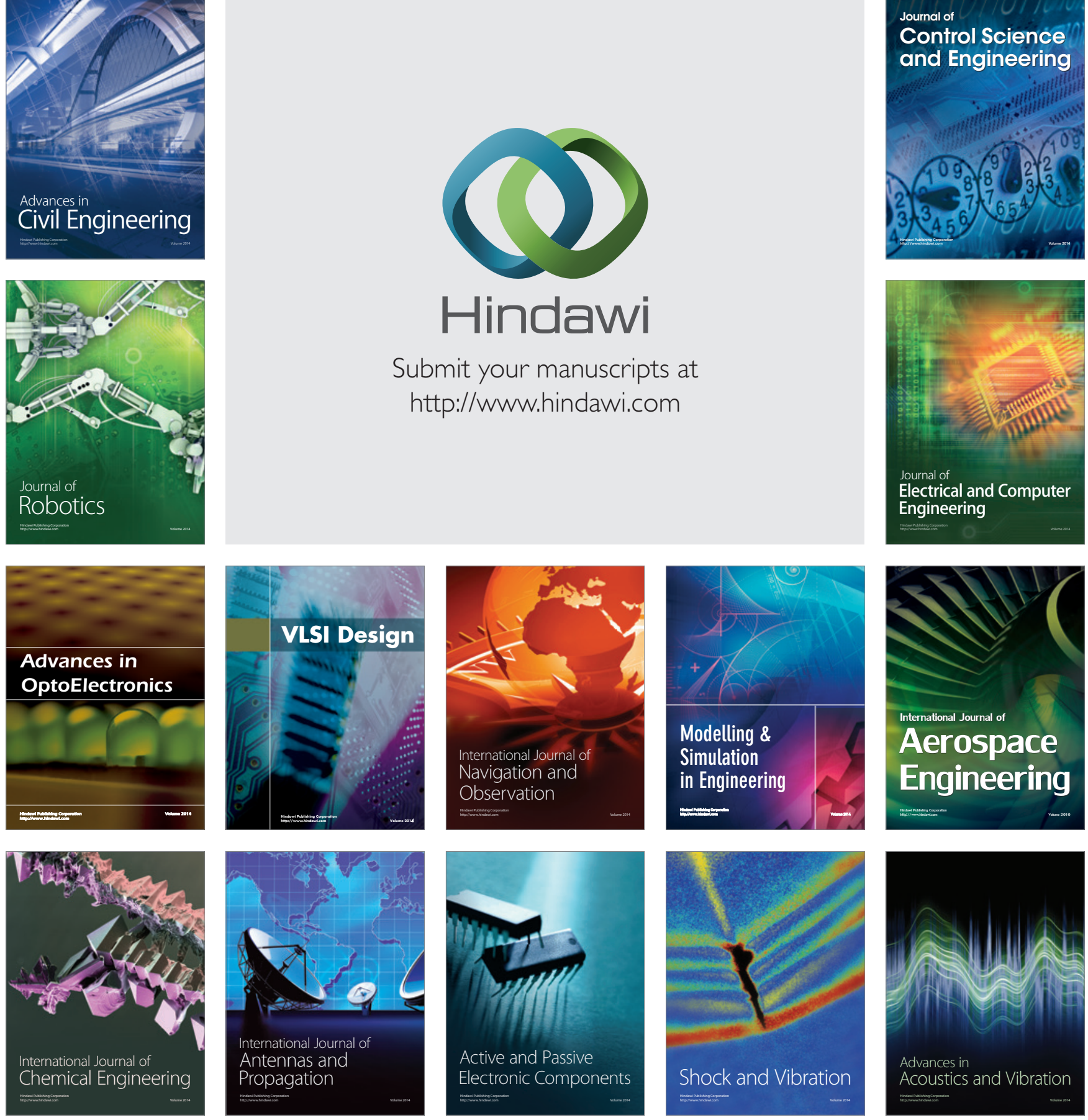